\title{
Georeference laser scanning data based on GNSS antennas models
}

\author{
Zofia Rzepecka, Mateusz Kowalski, Dariusz Tomaszewski \\ University of Warmia and Mazury in Olsztyn, Oczapowski Str. 1, Olsztyn 10-719, Poland
}

\begin{abstract}
Georeference of data coming from laser scanning is a relevant element of defining a cloud of points. Simultaneously, the process is timeconsuming according to a necessity of defining reference points coordinates. The authors of the article propose a method of a direct georeference of scanned data based on GNSS antennas' models. In the presented paper a theoretical attitude towards a problem was explained. This approach involves the use of GNSS antennas' scans and models. The results and derived conclusions are presented.
\end{abstract}

Keywords: laser scanning; georeference; GNSS antennas' models.

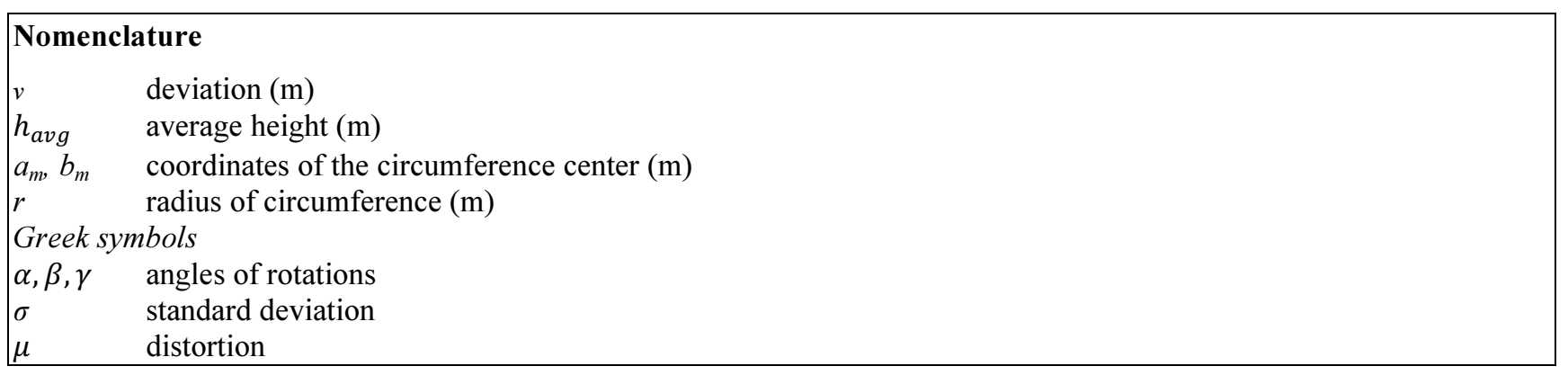

\section{Introduction}

Currently the major of the produced terrestrial laser scanners are not equipped with built in GPS/GNSS receivers. Due to that fact, the measurements require application of local coordinate system. Afterwards, a combination of well identified points in the cloud, reference points or surfaces are used to associate scans from different stations and their georeference concerning particular reference system. Most commonly reference spheres or aiming shields are used. Their patterns may differ depending on the producers brand. The basic characteristics of all used materials is high albedo allowing unambiguous identification of the target in the cloud of points. It is essential to determinate coordinates of reference points in admitted reference system configuration. Consequently, an additional measurement is required to determine their position in this system.

The authors of the article revised the possibilities of narrowing the procedure to set up GPS/GNSS antennas in the field. According to available geometric parameters (published by the NGS) of the antenna and the antenna's phase centre offsets as also points reflected from the antenna's side surface it is possible to determine geometric relation between the laser scanning data and the antenna's phase centre. If during the scanning works the GNSS receivers are switched on, it would make it possible to determine the antennas phase centers positions, identify them in the scan and complete transformation of the scanner data into the system accomplished by GNSS positioning. In case of using relative positioning based on ASGEUPOS POZGEO D service this is the frame PL-ETRF2000 or PL-ETRF89 [1].

Corresponding author: Dariusz Tomaszewski. E-mail address: dariusz.tomaszewski@uwm.edu.pl

http://dx.doi.org/10.3846/enviro.2014.243

(C) 2014 The Authors. Published by VGTU Press. This is an open-access article distributed under the terms of the Creative Commons Attribution License, which permits unrestricted use, distribution, and reproduction in any medium, provided the original author and source are credited. 


\section{The experiment}

The experiment aiming at georeferencing of laser scans basing on GPS antennas' models, was conducted on 25. 07.2013. The measurements were carried with:

- Laser scanner Leica ScanStation C10

- Total station Leica TS 30

- Ashtech Z-Xtreme receivers with Geodetic IV Rev A antennas

The measurements were carried at picket-stabilized stations. The GPS antennas were set on tripods above the measured points. Additional flat plates were assembled to them providing possibility of height reference. The first stage of the measurement consisted in 2 hour GPS static session. The interval of GPS signal recording was admitted as 10s and the elevation mask $15^{\circ}$. The GPS antennas were scanned twice during measurement time. The first measurement aimed at scanning the GPS antennas positioned above the reference points (pickets). The succeeding measurement was proceeded by scanning taped GPS antennas, that is antennas with a tape attached to its side surface. Street lamps were scanned as an independent control points. The next stage of the experiment was conducted after GPS observations. After closure of the session the GPS antennas were unhitched from tribrach and afterwards prisms were installed. The following step included a controlling tacheometric measurement to estimate distance from stations to reference points and control points. The set of the antennas' pictures and fragments of the obtained cloud of points of the scanned antenna are presented in Fig. 1.

(a)

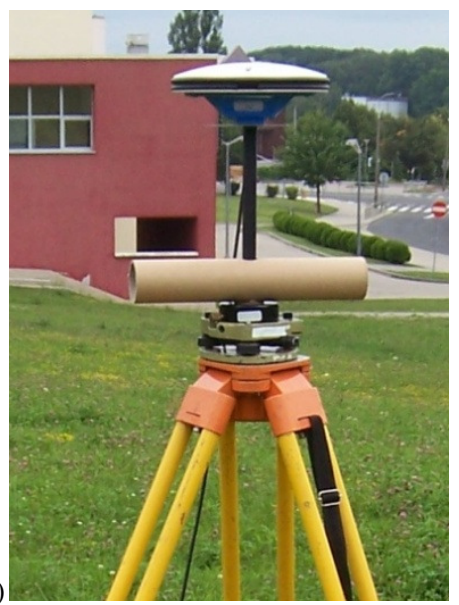

(b)

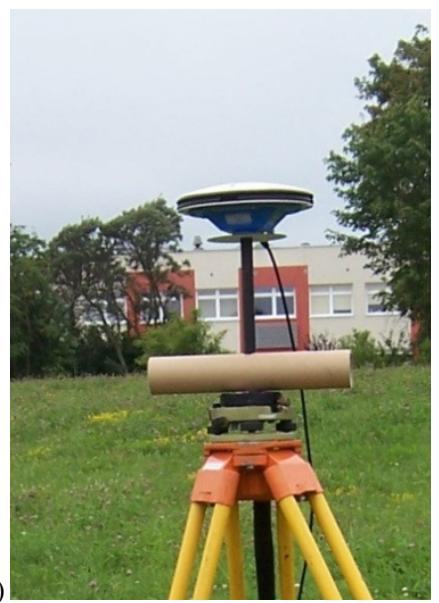

(c)

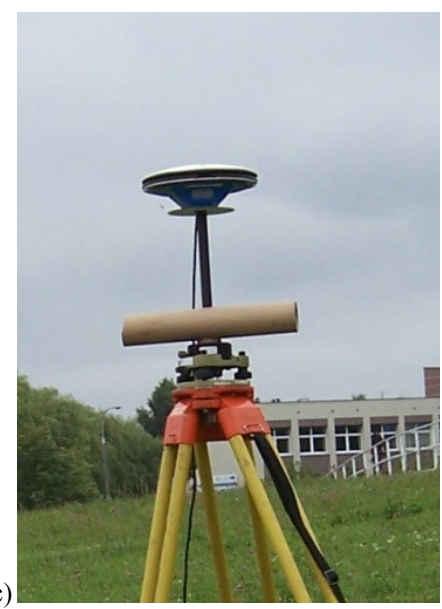

Fig. 1. GPS antennas pictures (a) antenna No 1 (b) antenna No 2 (c) antenna No 3

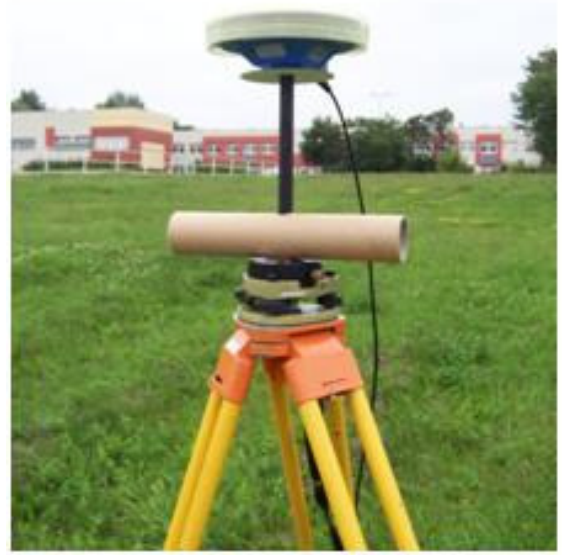

Fig. 2. Taped GPS antenna 
(a)

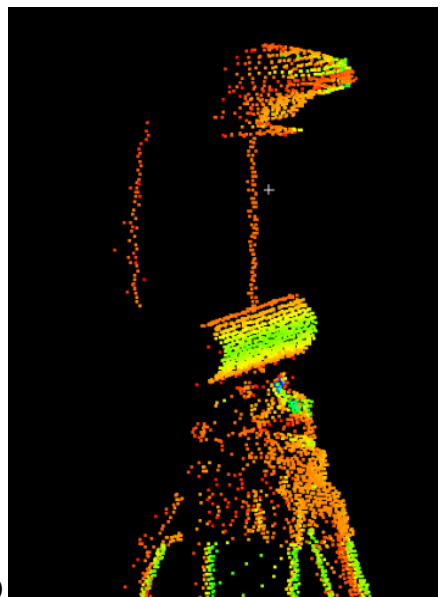

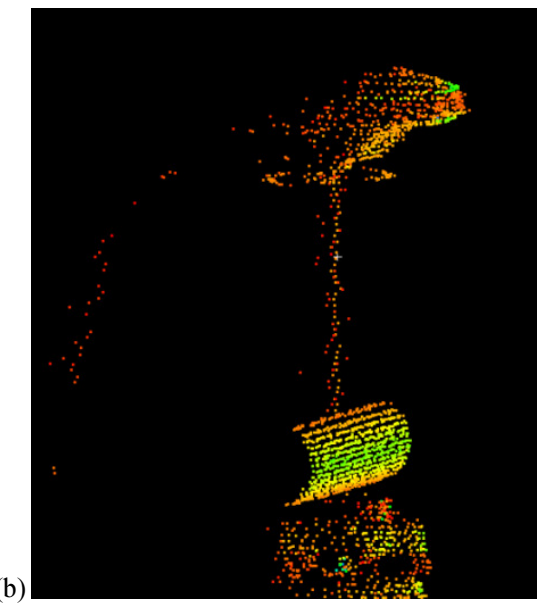

Fig. 3. Point cloud representing scanned (a) antenna No 1, (b) antenna No 2 and (c) antenna No 3
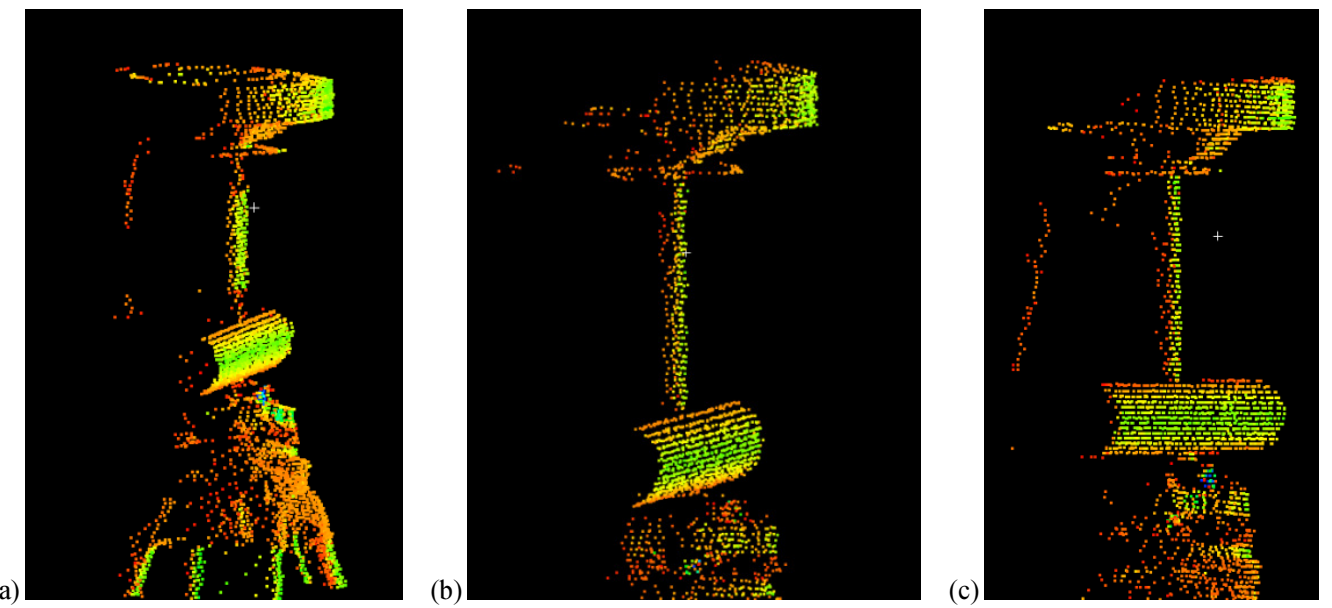

Fig. 4. Point cloud representing scanned (a) tapped antenna No 1 (b) tapped antenna No 2 (c) tapped antenna No 3

After checking the completeness of the obtained data, it was possible to perform the filtration of the cloud of points and its elaboration.

\section{Height fitting}

The height reference in this section means height in scanner local system. The height matching was performed by filtration of the cloud of points representing plate surface attached directly beneath the antennas and calculation of their arithmetical mean. The data was obtained by filtration:

- 28 points representing surface of the plate beneath antenna No 1

- 80 points representing surface of the plate beneath antenna No 2

- 70 points representing surface of the plate beneath antenna No 3

The average height, maximum deviations and standard deviations are presented in Table 1 . The scores were based on data elaboration and presented in meters.

Table 1. The antennas' heights

\begin{tabular}{lccc}
\hline & antenna nr 1 & antenna nr 2 & antenna nr 3 \\
\hline number of point & 28 & 80 & 70 \\
\hline$v_{\text {max }}$ & 0,001 & 0,004 & 0,004 \\
\hline$v_{\text {mini }}$ & $-0,001$ & $-0,005$ & $-0,003$ \\
\hline$\sigma$ & 0,001 & 0,002 & 0,002 \\
\hline$h_{\text {avg }}$ & 1,045 & 3,116 & 3,037 \\
\hline
\end{tabular}

The results are also graphically presented in Figure 5. 

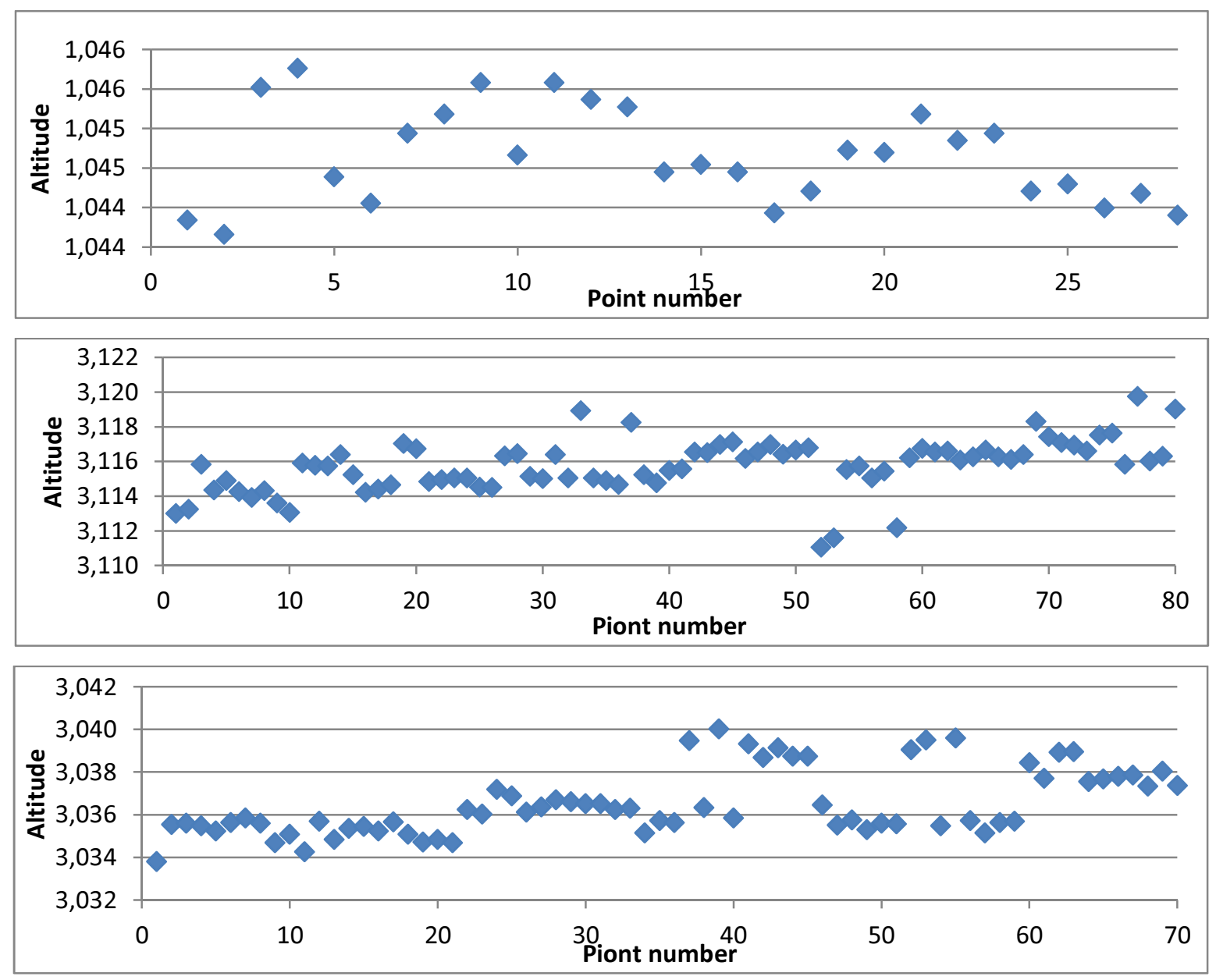

Fig. 5. Points representing points on the plate surface

To support lack of reflection of the points on the surface of the installed plates an additional horizontal pipes were assembled at vertical poles as showing in Fig. 6 .

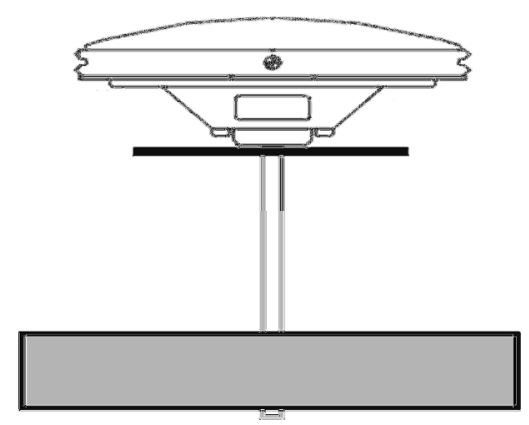

Fig. 6. Antenna's assembly model

In order to determine precisely height differences between antennas, a function "fit cylinder" from Leica Cyclone was used. As a result of matching cylinders to cloud of points, the following height differences were obtained.

Table 2. Height differences

\begin{tabular}{ll}
\hline & height difference \\
\hline $\mathrm{h} 1-\mathrm{h} 2$ & 2,072 \\
\hline $\mathrm{h} 1-\mathrm{h} 3$ & 1,993 \\
\hline $\mathrm{h} 3-\mathrm{h} 2$ & 0,080 \\
\hline
\end{tabular}




\section{Situational fitting}

The position matching was performed by placing circumferences in the cloud of points representing side surface of GPS antenna. Placing the circumferences defined as:

$$
(x-a)^{2}+(y-b)^{2}=r^{2}
$$

in the cloud of points $\left(x_{1}, y_{1}\right),\left(x_{2}, y_{2}\right), \ldots,\left(x_{n}, y_{n}\right)$ is possible by summing up squared distances between points and the centre of circumferences as defined below:

$$
S S(a, b, r)=\sum_{i=1}^{n}\left(r-\sqrt{\left(x_{i}-a\right)^{2}+\left(y_{i}-b\right)^{2}}\right)^{2}
$$

with known coordinates of the points in the circumference $\left(x_{i}, y_{i}\right)$ and coordinates of the centre of the circumference $(a, b)$ together with radius of the circumference $(r)$ assumed as unknown variables.

In the proposed method the circles were matched by modified method of the least squares [3]. Consequently, the minimum of the function was searched for:

$$
\operatorname{SSM}(a, b)=\sum_{i=1}^{n-1} \sum_{j=i+1}^{n}\left(a X_{j i}+b Y_{j i}-0,5\left(X_{i j}^{(2)}+Y_{i j}^{(2)}\right)\right)^{2}
$$

with derivates:

$$
\begin{gathered}
\frac{\partial S S M}{\partial a}=2 b \sum_{i=1}^{n-1} \sum_{j=i+1}^{n} X_{j i} Y_{j i}-\sum_{i=1}^{n-1} \sum_{j=i+1}^{n} X_{j i} Y_{j i}^{(2)}+2 a \sum_{i=1}^{n-1} \sum_{j=i+1}^{n} X_{j i}^{(2)}-\sum_{i=1}^{n-1} \sum_{j=i+1}^{n} X_{j i} X_{j i}^{(2)} \\
\frac{\partial S S M}{\partial b}=2 a \sum_{i=1}^{n-1} \sum_{j=i+1}^{n} Y_{j i} X_{j i}-\sum_{i=1}^{n-1} \sum_{j=i+1}^{n} Y_{j i} X_{j i}^{(2)}+2 b \sum_{i=1}^{n-1} \sum_{j=i+1}^{n} Y_{j i}^{(2)}-\sum_{i=1}^{n-1} \sum_{j=i+1}^{n} Y_{j i} Y_{j i}^{(2)}
\end{gathered}
$$

For each vector $\left(\alpha_{i}\right)$ and $\left(\beta_{i}\right)$ the following relation is hold:

$$
\sum_{i=1}^{n-1} \sum_{j=i+1}^{n}\left(\alpha_{j}-\alpha_{i}\right)\left(\beta_{j}-\beta_{i}\right)=n \sum_{i=1}^{n} \alpha_{i} \beta_{i}-\left(\sum_{i=1}^{n} \alpha_{i}\right)\left(\sum_{i=1}^{n} \beta_{i}\right)
$$

The coordinates of the circumference center are obtained when the derivates are compared to zero:

$$
\begin{aligned}
& a_{M}=\frac{D C-B E}{A C-B^{2}} \\
& b_{M}=\frac{A E-B D}{A C-B^{2}}
\end{aligned}
$$

where:

$$
\begin{aligned}
& a_{M}, b_{M}-\text { coordinates of the circumference center, } \\
& A=n \sum_{i=1}^{n} x_{i}^{2}-\left(\sum_{i=1}^{n} x_{i}\right)^{2}, \\
& B=n \sum_{i=1}^{n} x_{i} y_{i}-\left(\sum_{i=1}^{n} x_{i}\right)\left(\sum_{i=1}^{n} y_{i}\right), \\
& C=n \sum_{i=1}^{n} y_{i}^{2}-\left(\sum_{i=1}^{n} y_{i}\right)^{2}, \\
& \mathrm{D}=0,5\left(n \sum_{i=1}^{n} x_{i} y_{i}^{2}-\left(\sum_{i=1}^{n} x_{i}\right)\left(\sum_{i=1}^{n} y_{i}^{2}\right)+n \sum_{i=1}^{n} x_{i}^{3}-\left(\sum_{i=1}^{n} x_{i}\right)\left(\sum_{i=1}^{n} x_{i}^{2}\right),\right.
\end{aligned}
$$




$$
\mathrm{E}=0,5\left(n \sum_{i=1}^{n} y_{i} x_{i}^{2}-\left(\sum_{i=1}^{n} y_{i}\right)\left(\sum_{i=1}^{n} x_{i}^{2}\right)+n \sum_{i=1}^{n} y_{i}^{3}-\left(\sum_{i=1}^{n} y_{i}\right)\left(\sum_{i=1}^{n} y_{i}^{2}\right)\right)
$$

The calculation of the coordinates of the circumference's centre determined radius which constituted a comparison to actual radius of the antenna as declared by a producer. The radius of matched circumference is described [2]:

$$
r_{M}=\sum_{i=1}^{n} \frac{\sqrt{\left(x_{i}+a_{M}\right)^{2}+\left(y_{i}+b_{M}\right)^{2}}}{n}
$$

The results of the matched circles in the cloud of points representing a side surface of GPS antennas and taped side surface of GPS antenna were presented in the Tables 3 and 4 and Figures 7 and 8.

Table 3. Coordinates of the center and radius of the circumference

\begin{tabular}{lccc}
\hline & $a_{M}$ & $b_{M}$ & $r_{M}$ \\
\hline antenna No 1 & 0,006 & 20,542 & 0,097 \\
\hline antenna No 2 & $-20,669$ & 1,712 & 0,099 \\
\hline antenna No 3 & $-9,553$ & $-18,538$ & 0,099 \\
\hline
\end{tabular}

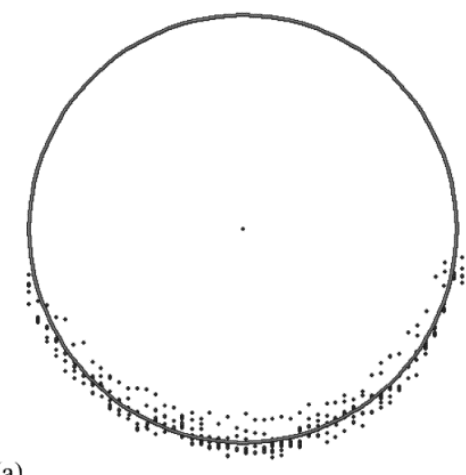

(a)

(b)

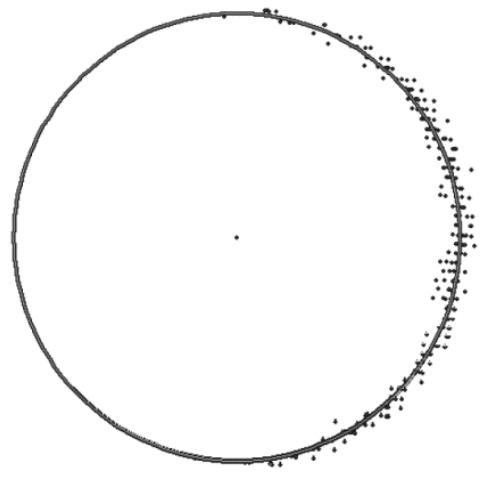

(c)

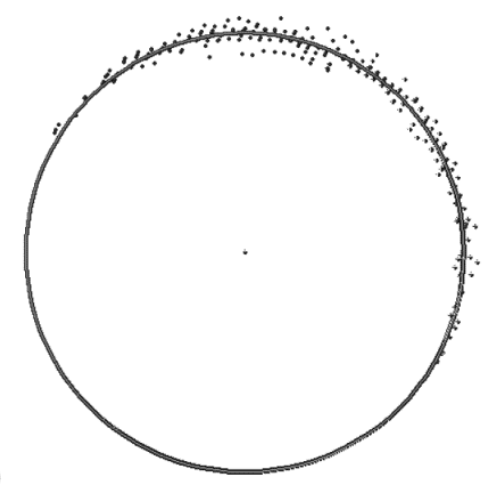

Fig. 7. Circle matched in Cloud of point (a) antenna No 1 (b) antenna No 2 (c) antenna No 3

Table 4. Coordinates of the center and radius of the circumference

\begin{tabular}{lrrl}
\hline & $a_{M}$ & \multicolumn{1}{c}{$b_{M}$} & \multicolumn{1}{c}{$r_{M}$} \\
\hline taped antenna No 1 & 0,006 & 20,545 & 0,103 \\
\hline taped antenna No 2 & $-20,670$ & 1,712 & 0,103 \\
\hline taped antenna No 3 & $-9,554$ & $-18,540$ & 0,103
\end{tabular}

(a)

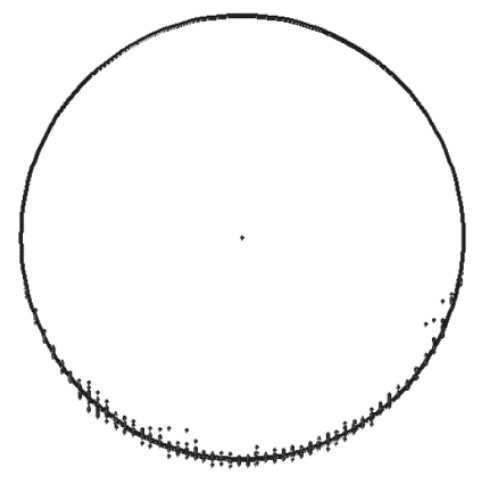

(b)

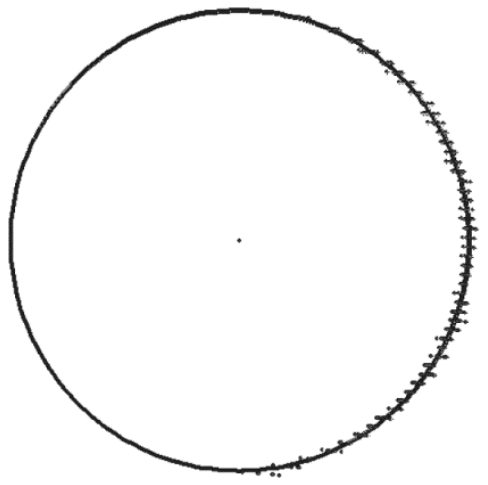

(c)

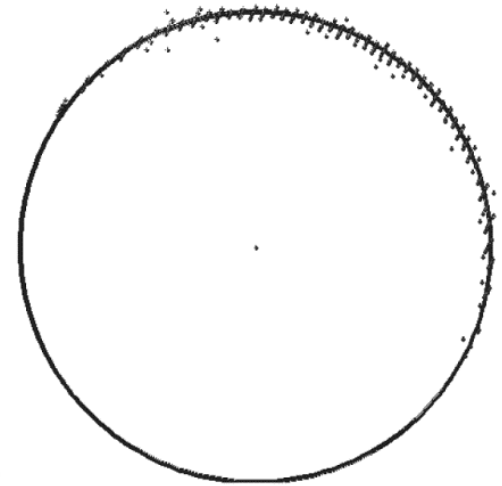

Fig. 8. Circle matched in Cloud of point (a) tapped antenna No 1 (b) tapped antenna No 2 (c) tapped antenna No 3

The taping of GPS antennas allowed flattening of the side surface of the antenna. Simultaneously, the scanned data is more homogenized which simplifies data filtering and accelerates process of circumference matching. 


\section{Results and analysis}

The results of comparison of the circumference matching both for taped and not taped antennas did not generate a significant difference in estimating antenna's phase centre. The standard deviations reach maximum $2 \mathrm{~mm}$. The application of additional horizontal pipes for extra height reference in case of lack of reflected point on the plates' surface was assessed as irrelevant. The sufficient number of points were reflected from the horizontal surfaces of the plates.

The potentiality of additional verification of height differences between antennas proved the validity of the method applied. The values of the height differences within multiplicity of methods were presented in the Table 5. The approximate values were reached also by calculating differences of coordinates. In this case the maximum standard deviations was $2 \mathrm{~mm}$.

Table 5. Coordinate differences

\begin{tabular}{lcc}
\hline & \multicolumn{2}{c}{ coordinates } \\
\cline { 2 - 3 } & $\mathrm{x}$ & $\mathrm{y}$ \\
\hline ant1 & 0,006 & 20,542 \\
\hline ant2 & $-20,669$ & 1,712 \\
\hline ant3 & $-9,553$ & $-18,538$ \\
\hline taped ant1 & 0,006 & 20,545 \\
\hline taped ant2 & $-20,670$ & 1,712 \\
\hline taped ant3 & $-9,554$ & $-18,540$ \\
\hline ant1 & 0,006 & 20,543 \\
\hline ant2 & $-20,669$ & 1,712 \\
\hline ant3 & $-9,554$ & $-18,539$ \\
\hline ant1 & 0,000 & 0,002 \\
\hline ant2 & 0,001 & 0,000 \\
\hline ant3 & 0,001 & 0,002 \\
\hline
\end{tabular}

Table 6. Height differences of vectors

\begin{tabular}{lllllll}
\hline \multirow{7}{*}{ vector } & \multicolumn{7}{c}{ height } \\
\cline { 2 - 7 } & flat plate & pipe & total station & GPS & $\begin{array}{l}\text { average height } \\
\text { difference }\end{array}$ & $\begin{array}{l}\text { standard } \\
\text { deviation }\end{array}$ \\
\hline $\mathrm{h} 1-\mathrm{h} 2$ & 2,071 & 2,073 & 2,072 & 2,071 & 2,072 & 0,001 \\
\hline $\mathrm{h} 1-\mathrm{h} 3$ & 1,992 & 1,993 & 1,991 & 1,992 & 1,992 & 0,001 \\
\hline $\mathrm{h} 3-\mathrm{h} 2$ & 0,079 & 0,079 & 0,080 & 0,079 & 0,080 & 0,001 \\
\hline
\end{tabular}

Table 7. Height differences of vectors

\begin{tabular}{llllllll}
\hline \multicolumn{7}{c}{ situational } \\
\hline \multirow{2}{*}{ vector } & $\begin{array}{l}\text { length of the vectors } \\
\text { lircle fitted into } \\
\text { GPS antenna }\end{array}$ & $\begin{array}{l}\text { circle fitted into GPS } \\
\text { antenna with tape }\end{array}$ & $\begin{array}{l}\text { total } \\
\text { station }\end{array}$ & GPS & $\begin{array}{l}\text { average } \\
\text { length }\end{array}$ & $\begin{array}{l}\text { standard } \\
\text { deviation }\end{array}$ \\
\hline xy1-xy2 & 27,964 & 27,967 & 27,965 & 27,965 & 27,965 & 0,001 \\
\hline xy1-xy3 & 40,232 & 40,237 & 40,235 & 40,235 & 40,235 & 0,002 \\
\hline xy3-xy2 & 23,101 & 23,103 & 23,102 & 23,102 & 23,102 & 0,001 \\
\hline
\end{tabular}

Table 8 . Slope length of the vectors

\begin{tabular}{lllllll}
\hline \multirow{2}{*}{ vector } & \multicolumn{5}{c}{ Slope length } \\
\cline { 2 - 7 } & flat plate & pipe & total station & GPS & $\begin{array}{l}\text { average } \\
\text { length }\end{array}$ & $\begin{array}{l}\text { standard } \\
\text { deviation }\end{array}$ \\
\hline ant1-ant2 & 28,041 & 28,041 & 28,041 & 28,043 & 28,042 & 0,001 \\
\hline ant1-ant3 & 40,281 & 40,281 & 40,284 & 40,282 & 40,282 & 0,001 \\
\hline ant3-ant2 & 23,101 & 23,101 & 23,102 & 23,104 & 23,102 & 0,002 \\
\hline
\end{tabular}


As formerly introduced, control objects were scanned and measured with usage of tacheometer. Three streetlights were chosen and measured. Afterwards, length differences between scanner station and streetlights were indicated. The results received from scanner and tacheometer were compared in the Table 9.

Table 9. Differences of length of the vectors

\begin{tabular}{|c|c|c|c|c|}
\hline \multicolumn{5}{|c|}{ control objects } \\
\hline \multirow{2}{*}{ vector } & \multicolumn{2}{|c|}{ length of the vectors } & \multirow{2}{*}{ average length } & \multirow{2}{*}{$\begin{array}{l}\text { standard } \\
\text { deviation }\end{array}$} \\
\hline & scanner & total station & & \\
\hline scanner-pole 1 & 40,012 & 40,004 & 40,008 & 0,005 \\
\hline scanner-pole2 & 56,405 & 56,423 & 56,414 & 0,013 \\
\hline scanner-pole3 & 80,317 & 80,351 & 80,334 & 0,024 \\
\hline
\end{tabular}

The cloud of points matched to determined coordinate system is a result of the experiment. The transformation from local/scanner coordinate system to the target coordinate system is possible due to the calculated coordinates of GPS antennas' centers in local coordinate system and coordinates from of GPS determinations. To perform transformation from the local scanner reference system to GPS (ASG-EUPOS) coordinate system, in the first step the computed GPS positions were presented in the local ENU system and then they were rotate to get small angles in the final 3D rotation matrix. The well-known approximation could be used and the transformation parameters were be computed from [4]:

$$
\left[\begin{array}{l}
x_{d} \\
y_{d} \\
z_{d}
\end{array}\right]=\left[\begin{array}{ccc}
m & \gamma & -\beta \\
-\gamma & m & \alpha \\
\beta & -\alpha & m
\end{array}\right] \cdot\left[\begin{array}{c}
x_{s} \\
y_{s} \\
z_{s}
\end{array}\right]+\left[\begin{array}{l}
x_{0} \\
y_{0} \\
z_{0}
\end{array}\right]
$$

where:

$m=1+\mu, \mu-$ distortion

$\alpha, \beta, \gamma-$ angles of rotations

$x_{d}, y_{d}, z_{d}$ - coordinates in the determined (target) coordinate system

$x_{s}, y_{s}, z_{s}$ - coordinates in local scanner system

$x_{0}, y_{0}, z_{0}$ - displacement vector

Transformation parameters are presented in Table 10. The results of transformation and standard deviations are in Table 11 .

Table 10. Transformation parameters

\begin{tabular}{rl}
\hline value & parameter \\
\hline $5,59733 \mathrm{E}-05$ & $\mu$ \\
\hline$-1,36044 \mathrm{E}-05$ & $\alpha$ \\
\hline$-7,90475 \mathrm{E}-06$ & $\beta$ \\
\hline$-1,18634 \mathrm{E}-05$ & $\gamma$ \\
\hline 0,000662642 & $x 0$ \\
\hline$-0,000799103$ & $y 0$ \\
\hline $7,61209 \mathrm{E}-05$ & $z 0$
\end{tabular}

Table 11. Calculated coordinates and model coordinates in determined coordinate system

\begin{tabular}{rrr}
\hline calculated coordinated & model coordinates & differences \\
\hline 0,0007 & 0,0000 & 0,0007 \\
\hline$-0,0008$ & 0,0000 & $-0,0008$ \\
\hline 0,0001 & 0,0000 & 0,0001 \\
\hline 26,8482 & 26,8500 & $-0,0018$ \\
\hline$-7,8348$ & $-7,8350$ & 0,0002 \\
\hline 2,0709 & 2,0710 & $-0,0001$ \\
\hline 38,3411 & 38,3400 & 0,0011 \\
\hline 12,2066 & 12,2060 & 0,0006 \\
\hline 1,9921 & 1,9920 & 0,0001 \\
\hline standard deviation & 0,0009
\end{tabular}




\section{Conclusions}

The results of the measurements prove the thesis of effective enforcement of georeference laser scanned data directly using GPS antennas. The suggested method does not imply time-consuming assembly of target or reference surface for georeference of the scanned data. The application of GPS measurements and GPS antennas' geometry (published by producers and by The International GNSS Service) the HDS scanned data can be effectively referred to the ASG-EUPOS reference system. The comparison of the vector length between antennas implies that horizontal installment of the additional cylindrical surface beneath the antenna is not required. The results received in plate measurement which was assembled under the antenna are sufficiently precise. The element is easily identifiable in the cloud of points. Furthermore, pinpointing its height on the base of scanned points of the surface is less time-consuming than matching cylinder into the points which represent a horizontal pipe. It is worth proceeding an analysis of describing optimum dimensions and material of the plate. The comparison of GPS antennas' coordinates and screen shots of the cloud of points proves that taping the antennas does not significantly impact the coordinates (differences less than $2 \mathrm{~mm}$ ). Nonetheless, the cloud of points from taped antenna presents a more regular reflection of the points and facilitates data filtration. Consequently, a usage of a regular shape antennas (side surface) or flattening side surface with tapes is advisable.

\section{References}

[1] Graszka, W. 2013. ASG-EUPOS Within the Applicable Technical Standards, in ASG-EUPOS Users Training, Olsztyn, Poland 2013.

[2] Coope, I. D. 1993. Circle fitting by linear and nonlinear least squares, Journal of Optimization Theory and Applications 76: $381-388$. http://dx.doi.org/10.1007/BF00939613

[3] Umbach, D. 2000. Jones, K.N. A Few Methods for Fitting Circles to Data, Transactions on instrumentation and measurement 20(Y).

[4] Vectors, Matrices and Coordinate Transformations [online]. 2009. Massachusetts Institute of Technology [cited 10 November 2013]. Available from Internet: http://ocw.mit.edu/courses/aeronautics-and-astronautics/16-07-dynamics-fall-2009. 